\title{
Surgical Strategies and Histopathologic Issues in the Management of Lentigo Maligna
}

\author{
Jonathan R. Stretch, MBBS, DPhil, FRACS ${ }^{1,2}$ and Richard A. Scolyer, MD, FRCPA, FRCPath ${ }^{1,3,4}$ \\ ${ }^{1}$ Sydney Melanoma Unit and Melanoma Institute Australia, Mater Hospital and Royal Prince Alfred Hospital, Sydney, \\ NSW, Australia; ${ }^{2}$ Discipline of Surgery, Faculty of Medicine, The University of Sydney, Sydney, NSW, Australia; \\ ${ }^{3}$ Discipline of Pathology, Faculty of Medicine, The University of Sydney, Sydney, NSW, Australia; ${ }^{4}$ Department of \\ Anatomical Pathology, Royal Prince Alfred Hospital, Camperdown, NSW, Australia
}

Lentigo maligna (LM) (also termed Hutchinson's melanotic freckle and precancerous melanosis of Dubreuilh) is a major clinical problem, particularly in individuals of European origin living in areas of high sunlight exposure, and its incidence continues to increase. LM is usually regarded as a form of melanoma-in-situ, although a distinction between different phases of LM as a melanoma precursor and in-situ melanoma has been proposed. ${ }^{1}$ The article by Moller et al. published in this issue highlights some of the important pathobiologic features of LM and the implications of its management. ${ }^{2}$ It also provides an appropriate opportunity to review some of the challenges associated with developing strategies to delineate and treat it.

Establishing a diagnosis of LM is often challenging for a number of reasons. First, it often shows overlapping clinical features with other benign lesions, and because of this, it may be misdiagnosed. Although a diagnosis of LM can be strongly suggested by clinical features, dermoscopy, and in vivo confocal microscopy, histopathology is usually considered the gold standard for diagnosis. ${ }^{3,4}$ Small (subtotal) biopsy samples are often taken in an attempt to verify the diagnosis before definitive treatment because the lesions are often located on the face and are important in size, location, or both. The diagnosis of LM is, however, frequently difficult for pathologists when interpreting these small biopsy specimens. There is often great heterogeneity in the histopathologic features of LM in different portions of the lesion. Parts of a LM may display histopathologic

(C) Society of Surgical Oncology 2009

Published Online: 26 March 2009

J. R. Stretch, MBBS, DPhil, FRACS

e-mail: jonathan.stretch@smu.org.au features of a benign lesion (such as a solar lentigo or dysplastic nevus), and therefore, performing small biopsies of areas that do not display the typical histopathologic features may lead to misdiagnosis. ${ }^{5}$ Furthermore, in its early stages, it may be difficult to histopathologically differentiate LM from melanocytic hyperplasia in sundamaged skin (so-called solar melanocytosis) because these processes represent a morphologic continuum. ${ }^{6-8}$

LM also represents a therapeutic challenge because of its usual size and location, and its propensity to locally recur. One of the particular difficulties encountered in LM is the diverse morphological spectrum by which it not infrequently manifests itself, including as a diffuse, poorly demarcated, and subtle process of melanocytic dysplasia, in contrast to the other, more discrete tumors managed in cutaneous oncology. In view of this, determination of the peripheral margins of LM clinically and pathologically represents another challenge, particularly because LM is often amelanotic peripherally and can extend far beyond the visible margins. Although some LMs can be relatively well defined clinically, and adjunctive techniques including dermoscopy, illumination with Wood's lamp, and in vivo confocal microscopy can all potentially aid in the identification of more subtle abnormal melanocytic proliferations, isolated abnormal melanocytes at the periphery of these lesions cannot always be reliably identified with these techniques. It is likely that unrecognized unresected qualitatively abnormal melanocytes beyond surgical resection margins are responsible for a high proportion of LM recurrences and potentially for occult progression to LM melanoma.

Patients with substantial accumulated solar exposure undoubtedly acquire a spectrum of genomic damage to melanocytes and the associated integumen in whole anatomic fields. This is evidenced indirectly by epidermal 
atrophy and solar elastosis in the dermis, which are characteristic features of LM, and more directly by recent genomic studies of these melanocytes. ${ }^{9-12}$ The question as to what constitutes appropriate clinical management and a satisfactory resection margin for LM therefore requires integration and resolution of multiple factors, including the patient's history, and particularly the clinical rate of evolution of the lesion, a semiqualitative histopathologic assessment of the severity of the primary melanocytic process (including at or near the peripheral margin), the patient's age and perceived risk of transformation into LM melanoma, the anatomic site (particularly the risk of functional impairment to adjacent structures especially on the face), and, importantly, an assessment of the transition of the melanocytic process from the lesion proper to the resection margin. In this regard, all the perimeter techniques for histopathologic assessment of surgical margins described to assist in defining the margin of LM have important limitations that should be recognized and considered. ${ }^{2,13-15}$ LM has a characteristic propensity to be associated with a tapering periphery characterized by a discontinuous proliferation of melanocytes, quantitatively increased numbers of isolated melanocytes, and isolated single melanocytes with demonstrable cytological atypia. Given that a large proportion of LMs are associated with peripheral features such as these, and that appreciable melanocytic changes can often be identified by separate remote sampling elsewhere in the anatomic field (related to chronic sun damage [solar melanocytosis]), the advantageous perspective gained by the pathologist examining radially oriented sections that display the transition, be it relatively circumscribed or an incrementally changing continuum, cannot be understated. The perimeter strip sampling methodology proposed by Moller et al. and others requires the pathologist to examine narrow bands of tissue oriented at $90^{\circ}$ to the radial axis from the center of the specimen and does not assist the pathologist in gaining a continuous perspective of the transitional lesion margin, which may complicate interpretation and even result in underdiagnosis or overdiagnosis of LM in some cases. ${ }^{2}$

We would therefore propose that clinicians involved in the surgical excision of LM carefully contemplate the underlying pathobiological changes in the melanocyte population within the larger anatomic territories of clinically apparent LMs and recognize the limitations of currently available techniques to define and delineate LM. The evolving genomic techniques used by Bastian et al. give some insight as to what may be needed to comprehensively assess the peripheral margin of these lesions, particularly in younger patients. ${ }^{16,17}$ His group has provided increasing evidence of the substantive genomic changes in field melanocytes (morphologically normal by light microscopy) located an appreciable distance away from the light microscopically identified margin of acral melanomas, and it is reasonable to expect that similar features will soon be described in association with LM. The fact that we currently contemplate treatment modalities such as radiotherapy and possibly topical agents such as imiquimod to treat the peripheral transitional areas surrounding clinically apparent LM highlights the shortcomings of current histological assessment and excisional strategies and tantalizes us with the prospect of a better treatment modality for a seemingly accessible neoplastic process that, with the proviso of adnexal involvement, is by definition superficial to the basement membrane of the dermo-epidermal junction. Until clinically applicable techniques are available to detect these occult partially transformed melanocytes at the periphery of LM, maintaining the histological perspective gained by histologically examining the excised lesion in continuous context with the surgical margin remains useful.

\section{REFERENCES}

1. Tannous ZS, Lerner LH, Duncan LM, et al. Progression to invasive melanoma from malignant melanoma in situ, lentigo maligna type. Hum Pathol. 2000;31:705-8.

2. Moller MG, Pappas-Politis E, Zager JS, et al. Surgical management of melanoma-in-situ using a staged marginal and central excision technique. Ann Surg Oncol. DOI:10.1245/s10434-0080239-x.

3. Stante M, Giorgi V, Stanganelli I, et al. Dermoscopy for early detection of facial lentigo maligna. Br J Dermatol. 2005;152: $361-4$.

4. Chen CS, Elias M, Busam K, et al. Multimodal in vivo optical imaging, including confocal microscopy, facilitates presurgical margin mapping for clinically complex lentigo maligna melanoma. Br J Dermatol. 2005;153:1031-6.

5. Dalton SR, Gardner TL, Libow LF, et al. Contiguous lesions in lentigo maligna. J Am Acad Dermatol. 2005;52:859-62.

6. Klauder JV, Beerman H. Melanotic freckle (Hutchinson), melanose circonscrite precancereuse (Dubreuilh). AMA Arch Dermatol. 1955;71:2-10.

7. Weyers W, Bonczkowitz M, Weyers I, et al. Melanoma in situ versus melanocytic hyperplasia in sun-damaged skin. Assessment of the significance of histopathologic criteria for differential diagnosis. Am J Dermatopathol. 1996;18:560-6.

8. Cohen LM. The starburst giant cell is useful for distinguishing lentigo maligna from photodamaged skin. J Am Acad Dermatol. 1996;35:962-8.

9. Saldanha G, Potter L, Daforno P, et al. Cutaneous melanoma subtypes show different BRAF and NRAS mutation frequencies. Clin Cancer Res. 2006;12:4499-505.

10. Akslen LA, Puntervoll H, Bachmann IM, et al. Mutation analysis of the EGFR-NRAS-BRAF pathway in melanomas from black Africans and other subgroups of cutaneous melanoma. Melanoma Res. 2008;18:29-35.

11. Lang J, MacKie RM. Prevalence of exon 15 BRAF mutations in primary melanoma of the superficial spreading, nodular, acral, and lentigo maligna subtypes. J Invest Dermatol. 2005;125:575-9.

12. Curtin JA, Fridlyand J, Kageshita T, et al. Distinct sets of genetic alterations in melanoma. N Engl J Med. 2005;353:2135-47.

13. Mahoney MH, Joseph M, Temple CL. The perimeter technique for lentigo maligna: an alternative to Mohs micrographic surgery. J Surg Oncol. 2005;91:120-5. 
14. Johnson TM, Headington JT, Baker SR, et al. Usefulness of the staged excision for lentigo maligna and lentigo maligna melanoma: the "square" procedure. J Am Acad Dermatol. 1997; 37:758-64.

15. Jeneby TT, Chang B, Bucky LP. Ultraviolet-assisted punch biopsy mapping for lentigo maligna melanoma. Ann Plast Surg. 2001;46:495-9.
16. Bastian BC, Kashani-Sabet M, Hamm H, et al. Gene amplifications characterize acral melanoma and permit the detection of occult tumor cells in the surrounding skin. Cancer Res. 2000;60: 1968-73.

17. North JP, Kageshita T, Pinkel D, et al. Distribution and significance of occult intraepidermal tumor cells surrounding primary melanoma. J Invest Dermatol. 2008;128:2024-30. 